\title{
ACOMPAÑAMIENTO FAMILIAR EN LA HOSPITALIZACIÓN DEL USUARIO PEDIÁTRICO DE 6 A 12 AÑOS
}

\author{
FAMILY SUPPORT DURING HOSPITALIZATION \\ OF THE 6 TO 12 YEARS OLD PEDIATRIC USER
}

\author{
Ángela Astudillo Araya* \\ Andrea Martínez Martínez ${ }^{* *}$ \\ Claudia Muñoz Badillo ${ }^{* * *}$ \\ Michelle Pacheco Lema ${ }^{* * *}$ \\ Ángela SEPÚlveda GaraY ${ }^{* * * *}$
}

\begin{abstract}
RESUMEN
Antecedentes. La hospitalización genera en el niño una adaptación al nuevo entorno, situación que produce estrés producto del cambio de ambiente, en donde la separación de sus padres de su entorno típico es un tema interesante de abordar para comprender el perfil del acompañante. Objetivo. Describir los tipos de acompañamiento familiar más frecuentes durante la hospitalización del usuario pediátrico de 6 a 12 años en el Centro de Costo Indiferenciado del Niño y la Niña (CCINN), del Complejo Asistencial Dr. Víctor Ríos Ruiz de la ciudad de Los Ángeles, Chile. Material y método. Estudio descriptivo de corte transversal, en el cual se seleccionó a 52 usuarios pediátricos entre 6 a 12 años hospitalizados en el CCINN del Complejo Asistencial Dr. Víctor Ríos Ruiz de Los Ángeles. Resultados. El 92,3\% de los padres y/o cuidadores que visitan a sus hijos están interesados en entender la situación actual del niño/a en su hospitalización y enfermedad (acompañamiento familiar tipo 1), mientras que un $7,7 \%$ de la muestra de padres con un esfuerzo disminuido (acompañamiento familiar tipo 2) $(\mathrm{z}=4,10 ; p<0,001)$, no registrando padres y/o cuidadores con conductas de bajo o escaso interés por la situación actual del niño/a que pudiera dificultar su integración frente a la situación de hospitalización y enfermedad (acompañamiento familiar tipo 3). Conclusión. Se logra evidenciar una preocupación de los padres y/o cuidadores por el proceso que vive el hijo y/o pupilo, en donde los profesionales pueden integrarse mediante la educación de técnicas o materias del cuidado posthospitalización del usuario pediátrico.
\end{abstract}

Palabras clave: Familia, apoyo, hospitalización, pediatría, atención al paciente.

\footnotetext{
ABSTRACT

Background. The hospital generates in the child adaptation to a new environment, a situation that produces stress because of the change, where the separation of their parents from their typical environment is an interesting topic to address in order to understand the profile of the accompanying person. Objective. To describe the types of most frequent family company observed during the hospitalization of the 6 to 12 years old pediatric user in the Child Undifferentiated Cost Center (UCCCh). Methods. Cross-sectional descriptive study, which selected 52 pediatric users aged 6 to 12, hospitalized in UCCCh at Dr. Víctor Ríos Ruiz Hospital, Los Ángeles, Chile. Results. $92.3 \%$ of parents and / or caregivers who visit their children are interested in understand the

* Enfermera. Académica Departamento de Enfermería. Facultad de Ciencias de la Salud y de los Alimentos. Universidad del Bío-Bío. Email: aastudil@ubiobio.cl

${ }^{* *}$ Enfermera. Hospital de Coelemu. Email: andre.ces@gmail.com

*** Enfermera Clínica Dávila. Email: Kallita88@hotmail.com

${ }^{* * * *}$ Enfermera. Hospital Clínico de Viña del Mar. Email: m.pacheco.lema@gmail.com

***** Enfermera. Email: ansepulgaray@gmail.com
} 
current situation of the child in his/her hospitalization and illness (family support type 1 ), while $7.7 \%$ of the sample shows declined efforts of parents (family support type 2$)(\mathrm{z}=4.10, \mathrm{P}<0.001)$, not registering parents / caregivers with a behavior that shows low or poor interest in the child's current situation / that could impede their integration addressing the situation of hospitalization and illness (family support type 3). Conclusion. It does demonstrate a concern from parents/caregivers through the process experienced by the child, where professionals can integrate them through education techniques or some care contents post-hospitalization for pediatric user.

Key words: Family, support, hospitalization, pediatrics, patient care.

Fecha recepción: 12/08/11 Fecha aceptación: 12/04/12

\section{INTRODUCCIÓN}

La hospitalización genera en el niño una adaptación al nuevo entorno, situación que produce estrés producto del cambio de ambiente, la separación de sus padres, familia y amigos y el dolor que puede producir la enfermedad; por otro lado se deben sumar las reacciones presentes en los padres, quienes se sienten incapaces de protegerlos cuando observan a sus hijos angustiados, irritables e inquietos o con alteraciones del sueño y apetito (1). Este usuario pediátrico que se encuentra en un ambiente ajeno y difícil es parte y centro de una familia, por lo que al abordar la hospitalización debe ser desde un contexto social familiar; porque es la familia, grupo social básico, quien debe moralmente proporcionar a sus miembros aspectos como seguridad, protección, socialización y compañía (2).

Los cambios realizados en el área de la salud se enfatizan en quienes deben proporcionar los cuidados durante y posterior a la enfermedad, principalmente los padres, quienes deben ser los máximos cuidadores de sus hijos, siendo responsables de los cuidados rutinarios, tales como alimentación, baño, estimulación del juego; situación que es natural cuando el niño está enfermo en el hogar. Por ello debe mantenerse aquel lazo durante la hospitalización, responsabilizándose de ciertos cuidados y cooperando con el equipo de salud. Así mismo los hospitales han permitido el acceso no sólo de los familiares, sino también de personas cercanas como: amigos, profesores y compañeros de curso, quienes también contribuyen a hacer más grato el ambiente hospitalario (3).

Enmarcado en el ámbito del cuidado del ser humano, se encuentra la "Teoría de los Cuidados" de Swanson (4); formulada empíricamente utilizando la metodología inductiva en investigaciones con mujeres que sufrieron pérdidas gestacionales, cuidados otorgados a los niños prematuros en la Unidad de Cuidados Intensivos Neonatales (UCIN) y en mujeres con riesgo social, se basa en cinco procesos básicos, que otorgan el significado a los actos etiquetados como cuidados. El primero corresponde al "conocimiento", definido como el esfuerzo para la comprensión del significado de un suceso en la vida del otro, evitando conjeturas, centrándose en la persona a la que se cuida, buscando claves, valorando meticulosamente y buscando un proceso de compromiso entre el que cuida y el que es cuidado. En segundo lugar, el "estar con", que se refiere a estar emocionalmente presente con el otro. Incluye estar allí en persona, transmitir disponibilidad y compartir sentimientos sin abrumar a la persona cuidada. En tercer término, se incluye el "hacer por", que significa hacer por otros lo que se haría para uno mismo, si fuera posible, incluyendo adelantarse a las necesidades, confortar, actuar con habilidad y competencia, y proteger al que es cuidado, respetando su dignidad. El cuarto componente es el "po- 
sibilitar", que corresponde a facilitar el paso del otro por las transiciones de la vida y los acontecimientos desconocidos, centrándose en el acontecimiento, informando, explicando, apoyando, dando validez a sentimientos, generando alternativas, pensando en las cosas detenidamente y dando retroalimentación. Por último, se encuentra el "mantener las creencias", que implica mantener la fe en la capacidad del otro de superar un acontecimiento o transición y enfrentarse al futuro con significado, creyendo en la capacidad del otro y teniéndolo en alta estima, manteniendo una actitud llena de esperanza, ofreciendo un optimismo realista, ayudando a encontrar el significado y estando al lado de la persona cuidada en cualquier situación (4).

En tal sentido, Chile se ha esmerado en otorgar un trato más amigable y grato a la comunidad, un ejemplo claro es lo comunicado por la Presidenta Michelle Bachelet en el discurso del 21 de mayo de 2006, donde se da a conocer la incorporación del Programa "Hospital Amigo", actualmente denominado "Hospital Amigo de la Familia y la Comunidad", iniciativa basada en los objetivos sanitarios 2006-2010 (5). En este contexto, el acompañamiento familiar lo reciben todos los usuarios pediátricos de 28 días a 15 años de edad, de parte de sus padres, un familiar y/o tutor; dichos acompañantes tienen derechos y deberes, tales como acceso a una sala de espera, casilleros para dejar sus objetos personales, derecho a ser informados por parte del médico de lunes a viernes en horario de 9 a 12 h y optar al beneficio de acompañamiento nocturno de 21:00 a 07:00 $h$ para lo cual deben identificarse con la enfermeras de turno, concluyéndose que el acompañamiento debe ser de forma periódica, ordenada y presente a lo largo de toda la hospitalización $(3,6,7)$.

Dado que la hospitalización es un proceso nuevo e inesperado, donde el usuario experimenta acontecimientos distintos a sus actividades diarias al igual que su cuidador, el objetivo de este trabajo es describir los tipos de acompañamiento familiar más frecuentes observados durante la hospitalización del usuario pediátrico de 6 a 12 años en el Centro de Costo Indiferenciado del Niño y la Niña (CCINN), del Complejo Asistencial Dr. Víctor Ríos Ruiz de la ciudad de Los Ángeles, Chile, con el objeto de contribuir en el entendimiento del acompañamiento como proceso vinculante entre el trabajo de recuperación de la salud y la familia.

\section{MATERIAL Y MÉTODOS}

Estudio descriptivo y corte transversal. La población del estudio correspondió a 52 usuarios pediátricos de 6 a 12 años hospitalizados en el CCINN del Complejo Asistencial Dr. Víctor Ríos Ruiz de la ciudad de Los Ángeles, Chile, quienes fueron seleccionados por un muestreo accidental entre el 9 de mayo al 12 de junio de 2010, a partir de los presentes al momento de aplicar los instrumentos y que cumplieran con los criterios de inclusión: estadía superior a una noche, que lo cuide la madre, padre, abuelo o cuidador legal mayor de 18 años al momento de aplicar la encuesta, alfabeto y que autorice firmando un consentimiento informado la aplicación de instrumentos. Se excluyeron a usuarios pediátricos que presenten dolor intenso, patologías crónicas invalidantes, enfermedades cognitivas o intelectuales como retardo mental, daño orgánico cerebral y síndrome de Down.

El levantamiento de los datos se realizó previa autorización, por el director del Complejo Asistencial Dr. Víctor Ríos Ruiz de Los Ángeles, previo estudio en el Comité de Ética de la Escuela de Enfermería de la Universidad del Bío-Bío. A cada familiar o tutor a cargo se le solicitó la participación del usuario pediátrico, explicando el objetivo, el carácter voluntario, no remunerado, confidencial y el aseguramiento que la aceptación o rechazo de la participación en el estudio no influiría 
en el tratamiento médico y/o de enfermería durante la estadía en el centro asistencial. Además, al usuario pediátrico se le otorgó un consentimiento verbal para participar de la investigación, en donde su negación hace inválida la firma del consentimiento informado por parte del adulto, en caso de que previamente lo haya aceptado.

Los datos fueron recolectados a través de dos instrumentos aplicados por las investigadoras a los usuarios pediátricos en el CCINN de Los Ángeles, y a sus respectivos acompañantes.

En primer lugar, se aplicó el Cuestionario Acompañamiento Familiar del Escolar Hospitalizado, confeccionado para esta ocasión, el que fue respondido por el acompañante del usuario pediátrico. Este cuestionario consta de 10 preguntas, de las cuales 4 son cerradas, 4 mixtas y 2 abiertas, que dan respuesta a las variables sociodemográficas del acompañante y variables relativas a la hospitalización.

Luego, a los usuarios pediátricos se les aplicó la Escala del Cuidado del Acompañante, modificado según la "Escala del Cuidado Profesional" de Swanson, que deriva de su Teoría de los Cuidados (8). El instrumento fue traducido al español por dos profesores de inglés y adaptadó al contexto pediátrico con palabras de mejor comprensión para el usuario pediátrico, posterior a esto, traducido nuevamente a su idioma original y enviado a la Dra. Swanson para su revisión y autorización. Presenta una escala tipo Likert que consta de 15 preguntas, formuladas en base a las cinco categorías básicas del cuidado planteadas en su teoría. Cada pregunta tiene 5 alternativas de respuestas; Si definitivamente (1 punto), En su mayoría (2 puntos), Más o menos (3 puntos), Ocasionalmente (4 puntos) y No en absoluto (5 puntos), además de la opción No se Aplica. En el contexto pediátrico la escala fue aplicada considerando sólo 13 preguntas, las dos restantes no fueron realizadas porque no se relacionaban con el estudio. Esta escala tiene un puntaje total de
65 puntos, los cuales son clasificados por las investigadoras en tres tipos de Acompañamiento Familiar según las cinco categorías básicas de la Teoría de los Cuidados.

En consecuencia, si el puntaje obtenido varía entre 1 y 21 puntos se consideró como Acompañamiento Familiar Tipo 1 (el acompañante está interesado en entender la situación actual del niño/a y cómo influye en su vida), entre 22 y 43 puntos se le catalogó como Acompañamiento Familiar tipo 2 (se observa un esfuerzo disminuido por comprender la experiencia vivida por el niño/a frente al proceso de hospitalización y enfermedad), mientras que si puntúa entre 44 a 65 puntos se le categorizó como Acompañamiento Familiar Tipo 3 (el acompañante presenta bajo o escaso interés por la situación actual del niño/a, dificultando su integración frente a la situación actual de hospitalización y enfermedad) (8).

Se procedió a aplicar los instrumentos en una muestra piloto de 10 usuarios pediátricos y sus respectivos acompañantes internados en el Servicio Médico-Quirúrgico (Cirugía Infantil) del Hospital Clínico Herminda Martín de Chillán, de modo que se verificó la comprensión de los instrumentos recolectores.

Se aplicaron frecuencias absolutas (n) y relativas porcentuales (\%), y se aplicó la prueba z-score para diferencias de proporciones. Se procesó la información con el programa estadístico InfoStat.

\section{RESULTADOS}

De los 52 usuarios pediátricos en estudio, la mitad de ellos fue de sexo femenino $(n=26)$, el 51,9\% estuvo dentro del rango de 6 a 8 años $(n=27)$ y el restante $48,1 \%$ presentó edades entre 9 y 12 años.

Respecto a los acompañantes (padres o cuidadores), la Tabla 1 muestra que la mayoría $(55,8 \%)$ presenta una edad entre los 
Tabla 1. Distribución de la muestra según características sociodemográficas del acompañante $(n=52)$.

\begin{tabular}{llcc}
\hline Variable & Categoría & Frecuencia & Porcentaje \\
\hline \multirow{3}{*}{ Edad } & Menor de 20 años & 0 & 0 \\
& 20 - 29 años & 13 & 25 \\
& $30-39$ años & 29 & 55,8 \\
& 40 o más años & 10 & 19,2 \\
\hline \multirow{2}{*}{ Sexo } & Femenino & 42 & 80,8 \\
& Masculino & 10 & 19,2 \\
\hline \multirow{3}{*}{ Parentesco } & Consanguíneo Primer Grado & 43 & 82,7 \\
& Consanguíneo Segundo Grado & 4 & 7,7 \\
& Consanguíneo Tercer Grado & 3 & 5,8 \\
& Ficticio & 1 & 1,9 \\
\multirow{2}{*}{ Procedencia } & Otros & 1 & 1,9 \\
& Urbano & 18 & 34,6 \\
\hline
\end{tabular}

30 a 39 años, el $80 \%$ es de sexo femenino, y una frecuencia importante de la muestra correspondió a personas con parentesco de primer grado respecto del usuario pediátrico (82,7\%). Por último, el 65,4\% de los acompañantes que constituyeron la muestra de estudio provinieron de zonas rurales vecindarias a la ciudad de Los Ángeles.

El 92,3\% presentó un perfil que la clasificó dentro de un acompañamiento familiar tipo $1(n=48)$, que corresponde a aquellos sujetos interesados en entender la situación actual del niño/a durante su hospitalización y enfermedad, y cómo influye en su vida, mientras que sólo 4 sujetos, que representan el 7,7\%, fueron clasificados en el grupo de acompañamiento tipo 2, definido como un esfuerzo disminuido de parte del acompañante por comprender la experiencia vivida por el niño/a frente al proceso de hospitalización y enfermedad, cifra significativamente menor en comparación a la proporción de padres y/o acompañantes clasificados como tipo $1(\mathrm{p}<0,001)$.
Respecto a las características personales del acompañante, la Figura 1 muestra que la mitad de la muestra la constituyeron sujetos considerados como acompañantes de tipo 1 cuyas edades fluctuaron entre 30 a 39 años de edad, casi el $77 \%$ fueron personas de sexo femenino y consanguinidad de primer grado y que provenían preferentemente de zonas rurales, en el 62\% de los casos. Respecto a los acompañantes catalogados como de tipo 2, el 5,8\% se concentró entre las edades de 30 a 39 años, no hay diferencia porcentual respecto al sexo (se observó una frecuencia de 3,9\% para hombres y mujeres). En cuanto al parentesco, se observa que preferentemente los acompañantes tipo 2 tienen consanguinidad de $2^{\circ}$ grado $(7,7 \%)$, y aquellos que provinieron de zonas urbanas es el mismo porcentaje de quienes lo hacen de zonas rurales.

En cuanto a la frecuencia de visitas, la Tabla 2 indica que casi la totalidad de los acompañantes visitan a sus hijos o familiares cercanos diariamente $(n=50)$. Respecto a 
Figura 1. Distribución de la muestra según características sociodemográficas del acompañante y tipo de acompañamiento $(n=52)$.

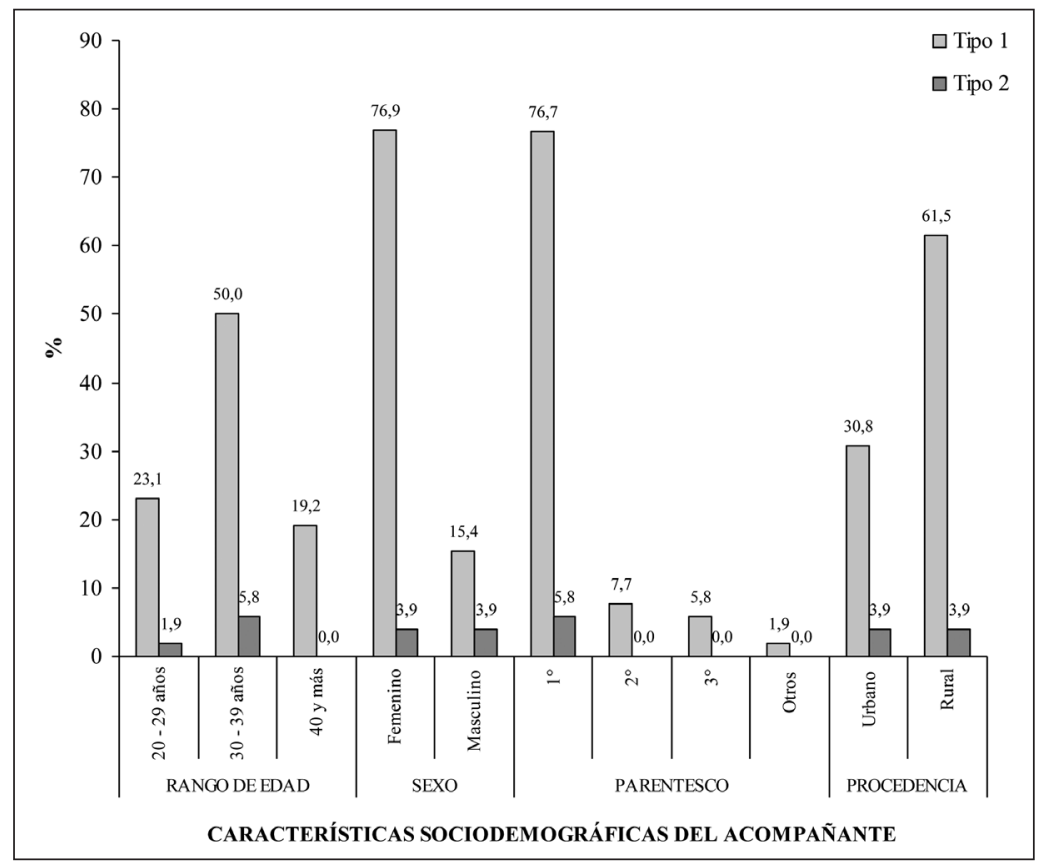

Tabla 2. Distribución de la muestra según factores de la hospitalización y tipos de acompañamiento $(\mathrm{n}=52)$.

\begin{tabular}{|c|c|c|c|c|c|c|c|}
\hline \multirow{2}{*}{ Variable } & \multirow{2}{*}{ Categoría } & \multirow{2}{*}{ n } & \multirow{2}{*}{$\%$} & \multicolumn{2}{|c|}{ Acompañamiento Tipo 1} & \multicolumn{2}{|c|}{ Acompañamiento Tipo 2} \\
\hline & & & & $\mathrm{n}$ & $\%$ & $\mathrm{n}$ & $\%$ \\
\hline \multirow{3}{*}{$\begin{array}{l}\text { Frecuencia } \\
\text { de visitas }\end{array}$} & Diario & 50 & 96,2 & 46 & 88,5 & 4 & 7,7 \\
\hline & Día por Medio & 1 & 1,9 & 1 & 1,9 & 0 & 0 \\
\hline & Cada dos o más días & 1 & 1,9 & 1 & 1,9 & 0 & 0 \\
\hline \multirow{3}{*}{$\begin{array}{c}\text { Cantidad de } \\
\text { horas } \\
\text { de visita }\end{array}$} & $1-8 h$ & 20 & 38,5 & 19 & 36,5 & 1 & 1,9 \\
\hline & $9-16 h$ & 18 & 34,6 & 16 & 30,8 & 2 & 3,9 \\
\hline & $17-24 h$ & 14 & 26,9 & 13 & 25 & 1 & 1,9 \\
\hline \multirow{2}{*}{$\begin{array}{l}\text { Acompañamiento } \\
\text { nocturno }\end{array}$} & Sí & 33 & 63,5 & 31 & 59,6 & 17 & 32,7 \\
\hline & No & 19 & 36,5 & 2 & 3,85 & 2 & 3,85 \\
\hline \multirow{2}{*}{$\begin{array}{l}\text { Experiencia Previa } \\
\text { de Hospitalización }\end{array}$} & Sí & 23 & 44,2 & 23 & 44,2 & 0 & 0 \\
\hline & No & 29 & 55,8 & 25 & 48,8 & 4 & 7,7 \\
\hline \multirow{5}{*}{$\begin{array}{l}\text { Actividades } \\
\text { de apoyo }\end{array}$} & Recreativas/ Educativas & 10 & 19,2 & 9 & 17,3 & 1 & 1,9 \\
\hline & Básicas/ de apoyo & 5 & 9,6 & 4 & 7,7 & 1 & 1,9 \\
\hline & Otras & 0 & 0 & 0 & 0 & 0 & 0 \\
\hline & $\begin{array}{l}\text { Recreativas/ Educativas y bá- } \\
\text { sicas vida diaria/ de Apoyo }\end{array}$ & 36 & 69,3 & 34 & 65,4 & 2 & 3,85 \\
\hline & Todas & 1 & 1,9 & 1 & 1,9 & 0 & 0 \\
\hline
\end{tabular}


las horas que dedican a la visita, el 38,5\% de los cuidadores realizó la visita en un intervalo de 1 a $8 \mathrm{~h}$ diarias $(\mathrm{n}=20)$, mientras que los acompañamientos que realizan entre 17 a $24 \mathrm{~h}$ en el día constituyen el 26,9\% ( $\mathrm{n}=14)$. Aproximadamente el 64\% de los padres y/o tutores realizan el acompañamiento durante las noches $(\mathrm{n}=33)$.

Cabe hacer notar que poco menos de la mitad de los usuarios pediátricos $(44,2 \%)$ tiene experiencias anteriores en el acompañamiento durante la hospitalización. Dentro de las actividades que realizan los acompañantes del usuario pediátrico durante su estadía como visitante destacan en primer lugar actividades recreativas, educativas y básicas de la vida diaria y de apoyo $(69,3 \%)$, seguido de sólo actividades recreativas (19,2\%), seguido muy detrás de aquellos acompañantes que realizan sólo actividades básicas de apoyo $(9,6 \%)$.

Si se estudia la distribución según el tipo de acompañamiento de los padres y/o tutores a cargo del menor, quienes son los interesados en entender la situación actual del niño/a durante su hospitalización, se obtuvo que el acompañamiento más óptimo visita a su usuario pediátrico a diario o día por medio (tipo 1) fue de casi el $90 \%$ ( $\mathrm{n}=47$ ), el $25 \%$ de ellos realiza un acompañamiento con una duración entre 17 a 24 h, casi el $60 \%$ realiza visitas durante la noche $(n=17)$ y el $65,4 \%$ realiza en conjunto actividades recreativas, educativas, básicas de la vida diaria y de apoyo al niño $(n=34)$. De este mismo grupo, casi la mitad de la muestra indicó que el usuario pediátrico no tenía experiencias previas de hospitalización $(\mathrm{n}=25)$.

\section{DISCUSIÓN Y COMENTARIO}

El proceso de enfermedad entendido como: "Pérdida de salud, alteración o desviación del estado fisiológico en una o varias partes del cuerpo, de etiología en general conocida, que se manifiesta por síntomas y signos característicos y cuya evolución es más o menos previsible" (9), aborda al usuario de forma biopsicosocial, explicando que existen repercusiones físicas y psicológicas, requiriendo en algunas circunstancias de hospitalización, entendida como: "Período de tiempo que se necesita para el restablecimiento de la salud óptima, tanto física como psicológica, en el que es necesario permanecer recluido en un centro de salud, hospital o clínica, con el fin de recibir los tratamientos y cuidados adecuados" (10).

Existen diversos acercamientos al tema en desarrollo; siendo Guerrero y Gamboa (11), quienes indican que se debe incorporar de forma más activa a los padres en el proceso de hospitalización, ya que influye positivamente en el desarrollo psicosocial y cognitivo del niño, favoreciendo la recuperación de los usuarios hospitalizados; ello coincide con la revisión bibliográfica realizada por Harrisson (12) que confirma la importancia de la atención centrada en la familia, reflejando como puntos importantes el apoyo y la participación de los padres en los cuidados y diversas actividades que éste conlleva. Ello es similar a lo expresado por Roberts (13), el cual señala que los padres durante el proceso de hospitalización disminuyen el llanto y sueño de sus hijos, fomentan la estimulación; de caso contrario, en niños no acompañados se observan tristes y estresados.

Conjunto a la favorable incorporación de los padres dentro del ambiente hospitalario y el proceso de hospitalización se encuentra el estudio de Tiedeman (14) que indica diversos aspectos que aumentan el nivel de ansiedad en los padres como son: demandas de la enfermedad, desconocimiento del medio ambiente, cambio de roles de los padres y la búsqueda de satisfacer necesidades de sus hijos; principalmente frente al dolor, incomodidad y procedimientos invasivos; congruente con la favorable incorporación de los padres a la hospitalización durante los últimos años y principalmente en actividades diarias a desarrollar durante este proceso 
por el interés que los padres expresan desde el inicio de la hospitalización.

Relacionado directamente con el área en estudio se encuentra el trabajo de López de Dicastillo y Cheung (15), que señala que un 97.4\% de los progenitores participantes eran mujeres, con rango etario predominante de 30 a 40 años en un 70\% del total, e indica que el $98 \%$ de los padres desean participar en las actividades derivadas del ingreso hospitalario, subdividiéndose de la siguiente forma: $41 \%$ cuidados básicos, $37 \%$ administración de medicamentos y $23 \%$ otras actividades enfermeras.

El estudio de Alfaro y Atria (10) revela que el desarrollo socioemocional del niño permite un mayor grado de resiliencia frente al proceso de hospitalización y un mal ambiente hospitalario influye negativamente en este proceso; es por esto que se debe adecuar el ambiente hospitalario, haciéndolo más grato hacia el niño.

Otra área importante durante la hospitalización, como es la recreación, entrega múltiples beneficios para el usuario pediátrico, lo que incide en gran medida en el desarrollo armónico de su personalidad; logrando tener una mejor valoración de la hospitalización, que será positiva si las actividades son desarrolladas por sus familiares, estimulando su interés visual (dibujos, juguetes, fotografías), auditivo (música, risas, voces), olfativo (perfumes) y táctil (madera plástico, textiles) (16), idea que enlaza lo indicado por Wilson et al. (17), donde además de avalar la creciente inclusión de los padres en el proceso de hospitalización disminuyendo las restricciones que existían para éstos, comenta los cambios ambientales en los centros de hospitalización, atendiendo no sólo necesidades físicas, sino aquellas que incorporan computadores, películas, juegos y otras actividades. Además este estudio reveló que los niños al encontrarse solos se encontraban temerosos, enojados, asustados y tristes, querían estar acompañados y recibir protección; y se molestaban al ver que sus padres no estaban allí.
Además se debe tener presente, como indica el estudio de Clatworthy et al. (18), que las actividades desarrolladas por el usuario pediátrico, por ejemplo las catalogadas como lúdicas, son herramientas de medición de la ansiedad del proceso en curso, por lo que deben ser fomentadas en circunstancias nuevas y complejas como es la hospitalización.

Claramente el tipo de acompañante que prevalece según lo expresado por los usuarios pediátricos fue el clasificado como tipo 1 , quienes tienen características positivas para afrontar y facilitar el proceso de hospitalización, entre ellas interés de comprender y compartir el proceso tanto en el ámbito físico y emocional, favoreciendo la expresión de sentimientos por parte del usuario pediátrico, ser parte de ciertas actividades propias de la hospitalización y además de favorecer la creencia en él, superar el proceso y en su esperanza de recuperación.

Este estudio es único en valorar la opinión de los escolares hospitalizados referente de su acompañante principal; y deja en evidencia que se requieren más investigaciones centradas en el punto de vista del usuario pediátrico para confirmar estos resultados y además mejorar las intervenciones, que han avanzado durante los últimos años, información útil para el equipo de salud, mejorando lo que ya se ha implementado y para los acompañantes, conociendo cómo debe ser su participación.

Dado que con mayor frecuencia se observó a familiares interesados en entender la situación actual del niño/a y cómo influye en su vida, visitarlo diariamente entre 1 y $8 \mathrm{~h}$, con acompañamiento nocturno, sin experiencia previa de hospitalización y cuyo contenido predominante de las visitas fueron las relacionadas con recreación, educación, actividades de la vida diaria y de apoyo, el rol del Profesional de Enfermería debe estar enfocado a la compenetración activa con el familiar para que se convierta en un coadyuvante fundamental en el proceso de recuperación del paciente. 


\section{REFERENCIAS}

1. Bausa L. Características y déficit inherentes a la hospitalización infantil. Docencia e Investigación [Internet]. 2002 Ene-Dic [citado 17 agosto 2009]; 2. Disponible en: http://www.uclm.es/varios/revistas/docenciaeinvestigacion/numero2/luisrodriguez.asp

2. Instituto interamericano del niño. Concepto de familia [Internet]. Montevideo: Instituto interamericano del niño; [sf] [citado 12 agosto 2009]. Disponible en: http://www.iin.oea.org/Cursos_a_distancia/Lectura\%2012_UT_1.PDF

3. Valdez Sánchez CA, Flórez Lozano JA. El niño ante el hospital: Programas para reducir la ansiedad hospitalaria. España: Ed. Universidad de Oviedo; 1995. p 61, 65; 132-133; 144; 158-159; 166-168; 185.

4. Swanson KM. Empirical development of a middle range theory of caring. Nurs Res. 1991; 40(3): 161-166.

5. Gobierno de Chile, Ministerio de Salud. Orientaciones Hospital amigo. [Internet]. Santiago, Chile: Subsecretaría de redes asistenciales; 2007 [citado 12 agosto 2009]. Disponible en:http://www.ssmso. $\mathrm{cl}$

6. Centro de Costo Indiferenciado del Niño y la Niña Complejo Hospitalario "Dr. Víctor Ríos Ruiz". Estrategia Hospital amigo de la familia y la comunidad. [Internet] Los Ángeles, Chile [sf] [citado 14 mayo 2010]. Disponible en: http://www. hospitallosangeles.cl/infor/pacientes/pediatria.pdf

7. Alonso-López J. Programa de Preparación Psicológica en Cirugía Infantil Programada. Revista Profesional Española de Terapia Cognitivo-Conductual 2005; 3(1): 56-70.

8. Swanson, K. Caring Professional Scale. En: Watson, editor. Assessing and Measuring Caring in Nursing and Health Scien- ce. Washington: Springer Publishing Company; 2002. p. 203-2006.

9. Diccionario Terminológico de Ciencias Médicas [Internet] 13a ed. España: Masson; 1999. Qué es salud y enfermedad: definiciones [citado 17 agosto 2009]. Disponible en: http://saludbio.com/articulo/salud-y-enfermedad-definicionespuntos-de-vista-diferentes

10. Alfaro A, Atria R. Factores ambientales y su incidencia en la experiencia emocional del niño Hospitalizado Santiago, Chile. Revista Pediatría Electrónica 2009; 6 (1): 36-54.

11. Guerrero, Gamboa S. Cuidado del niño hospitalizado con apoyo familiar. Avances de Enfermería 2002; 2: 23-32.

12. Harrisson TM. Family-Centered Pediatric Nursing Care: State of the Science. J Pediatr Nurs. 2010; 25(5): 335-343.

13. Roberts CA. Unaccompanied Hospitalized Children: A Review of the literature and incidence study. J Pediatr Nurs. 2010; 25(6): 470-476.

14. Tiedeman ME. Anxiety Responses of Parents During and After the Hospitalization of Their 5-to 11-Year-Old Children. J Pediatr Nurs. 1997; 12(2): 110-9.

15. López de Dicastillo O, Cheung Ph. Enfermería infantil y los cuidados centrados en la familia. Enferm Clin. 2004; 14(2): 83-92.

16. Fundación Anna Vázquez. Soporte emocional del niño hospitalizado. [Internet] Argentina. [sf] [citado 12 diciembre 2009]. Disponible en: http://fundacionannavazquez.wordpress.com

17. Wilson M, Megel M, Enenbach L, Carlson K. The Voices of Children: Stories About Hospitalization. J Pediatr Health Care. 2010; 24(2): 95-102.

18. Clatworthy et al. Child Drawing: Hospital-An Instrument Designed to Measure the Emotional Status of Hospitalized School-Age Children. J Pediatr Nurs. 1999; 14(1): 2-9. 